\title{
REGULARITY EQUIVALENCE OF THE SZEGÖ PROJECTION AND THE COMPLEX GREEN OPERATOR
}

\author{
PHILLIP S. HARRINGTON, MARCO M. PELOSO, AND ANDREW S. RAICH
}

(Communicated by Franc Forstneric)

\begin{abstract}
In this paper we prove that on a CR manifold of hypersurface type that satisfies the weak $Y(q)$ condition, the complex Green operator $G_{q}$ is exactly (globally) regular if and only if the Szegö projections $S_{q-1}, S_{q}$ and a third orthogonal projection $S_{q+1}^{\prime}$ are exactly (globally) regular. The projection $S_{q+1}^{\prime}$ is closely related to the Szegö projection $S_{q+1}$ and actually coincides with it if the space of harmonic $(0, q+1)$-forms is trivial.

This result extends the important and by now classical result by $\mathrm{H}$. Boas and E. Straube on the equivalence of the regularity of the $\bar{\partial}$-Neumann operator and the Bergman projections on a smoothly bounded pseudoconvex domain.

We also prove an extension of this result to the case of bounded smooth domains satisfying the weak $Z(q)$ condition on a Stein manifold.
\end{abstract}

\section{INTRODUCTION}

The goal of this article is to discuss the general principle that the combination of an appropriate weighted theory, a Hodge decomposition, and the $L^{2}$ regularity of $\bar{\partial}_{b}$ (resp., $\bar{\partial}$ ) provides the tools to prove the equivalence of regularity in the Sobolev scale between the complex Green operator (resp., the $\bar{\partial}$-Neumann operator) and the Szegö projection (resp., the Bergman projection).

H. Boas and E. Straube first observed the equivalence of the regularity of the Bergman projection and the $\bar{\partial}$-Neumann operator on smooth, bounded pseudoconvex domains in $\mathbb{C}^{n}$. In BS90 they proved the following theorem.

Theorem (Boas and Straube). Let $\Omega$ be a smooth, bounded pseudoconvex domain in $\mathbb{C}^{n}$. Let $1 \leq q \leq n$. Then the $\bar{\partial}$-Neumann operator $N_{q}$ on $(0, q)$-forms is exactly regular if and only if the three Bergman projections $P_{q-1}, P_{q}$, and $P_{q+1}$ are exactly regular.

The corresponding statement holds with the words "exactly regular" replaced by the words "globally regular".

Received by the editors May 1, 2013.

2010 Mathematics Subject Classification. Primary 32W10, 32W05, 35N15, 32V20, 32Q28.

Key words and phrases. $\bar{\partial}_{b}$, close range, Kohn's weighted theory, CR manifold, hypersurface type, tangential Cauchy-Riemann operator, $Y(q)$, weak $Y(q), Z(q)$, weak $Z(q)$, complex Green operator, $\bar{\partial}$-Neumann operator, Stein manifolds.

The first author was partially supported by NSF grant DMS-1002332.

This paper was written while the second author was visiting the University of Arkansas. He wishes to thank this institution for its hospitality and for providing a very pleasant working environment.

The third author was partially supported by NSF grant DMS-0855822. 
Recall that an operator is exactly regular if it preserves all $L^{2}$ Sobolev spaces and is globally regular if it preserves $C^{\infty}$ functions (or forms).

In this paper in particular we address the question of whether such a theorem has a counterpart in the case of the Szegö projection and the complex Green operator (see Sections 1 and 2 for precise definitions). One of the main results of this paper contains the following theorem as a special case.

Theorem. Let $\Omega$ be a smooth, bounded pseudoconvex domain in $\mathbb{C}^{n}$ and let $M$ denote its boundary. Let $G_{q}$ denote the complex Green operator and $S_{q}$ the Szegö projection on $(0, q)$-forms on $M, 1 \leq q \leq n-2$. Then the operator $G_{q}$ is exactly regular if and only if the three Szegö projections $S_{q-1}, S_{q}$, and $S_{q+1}$ are exactly regular.

The corresponding statement holds with the words "exactly regular" replaced by the words "globally regular".

Specifically, in this paper we study the cases of the complex Green operator $G_{q}$ on embedded CR manifolds of hypersurface type that satisfy the weak $Y(q)$ condition and the $\bar{\partial}$-Neumann operator on domains in a Stein manifold that satisfy the weak $Z(q)$ condition.

The required estimates and weighted theory are proven by the first and third authors in [HR11] and [HR], respectively, and the results in this article can be thought of as a consequence of the techniques of [BS90] and the estimates in [HR11, $\mathrm{HR}$.

We write the paper from the point of view of CR manifolds of hypersurface type and only indicate the changes that are needed to obtain the results for the $\bar{\partial}$-Neumann operator on weakly $Z(q)$ domains in Stein manifolds.

Let $M^{2 n-1} \subseteq \mathbb{C}^{N}$ be a $C^{\infty}$ compact, orientable CR manifold $N \geq n$. We say that $M$ is of hypersurface type if the CR-dimension of $M$ is $n-1$ so that the complex tangent bundle of $M$ splits into a complex subbundle and one totally real direction. The $\bar{\partial}_{b}$-complex on $M$ is obtained by restricting the de Rham complex on $M$ to the conjugate of the complexification of the complex subbundle.

When $M$ is the boundary of a pseudoconvex domain, closed range of $\bar{\partial}_{b}$ on $L_{p, q}^{2}(M)$ for $0 \leq p \leq n$ and $0 \leq q \leq n-1$ was proved by Shaw and Boas-Shaw Sha85, BS86. Independently, Kohn also proved closed range for $\bar{\partial}_{b}$ at all form levels and established the weighted theory in Koh86]. Nicoara generalized Kohn's results to the case of CR manifolds of hypersurface type $M$ when $\operatorname{dim}_{\mathbb{R}} M \geq 5$ Nic06]; see Baracco Bar12b, Bar12a for the case $\operatorname{dim}_{\mathbb{R}} M=3$. Harrington and Raich further generalized Nic06 by investigating closed range and the weighted theory for $\bar{\partial}_{b}$ on $(0, q)$-forms for a fixed $q$ (in this case, $p$ is irrelevant and they take $p=0$ for simplicity). They called their condition weak $Y(q)$ and developed the most general version of it in $[\mathrm{HR}$. Condition $Y(q)$ is well known to be the natural generalization of strict pseudoconvexity for dealing with $(0, q)$-forms on $M$ for a fixed $q$. See also ABZ06, Zam08, for conditions related to, but stronger than, weak $Y(q)$.

The paper concludes with a discussion of how to adapt the argument for the $\bar{\partial}$-Neumann operator and Bergman projection on a smooth, bounded domain in a Stein manifold. The argument follows the general argument for the complex Green operator and Szegö projection with some minor (and well-known) modifications. 
Harrington and Raich [HR] develop the $L^{2}$ and weighted Sobolev theory (for $-\frac{1}{2} \leq$ $s \leq 1)$ under the hypotheses that $\Omega \subset M$ is $C^{3}$, bounded, and satisfies weak $Z(q)$. In this paper, we discuss the generalization of the weighted theory for $s \geq 1$ when $\Omega$ is smooth and bounded. The $L^{2}$ and weighted $L^{2}$ theories for $\bar{\partial}$ on pseudoconvex domains in Stein manifolds are now classical and were established by Hörmander Hör65 and Kohn Koh73.

The outline of the paper is as follows. We set our notation in Section 1, state the main results in Section 2, and prove our results in Section 3, We conclude with a discussion of Stein manifolds in Section 4 .

\section{Notation}

Throughout the paper, we denote by $M$ a smooth, compact, embedded and orientable CR manifold of dimension $2 n-1$ and hypersurface type. We refer to Bog91 for the theory of CR manifolds.

1.1. The Levi form and weak $Y(q)$. Let $T^{p, q}(M)$ denote the collection of $(p, q)$ vectors and $\Lambda^{p, q}(M)$ the set of $(p, q)$-forms on $M$. The induced CR-structure has a local orthonormal basis $L_{1}, \ldots, L_{n-1}$ for the $(1,0)$-vector fields in a neighborhood $U$ of each point $x \in M$. Let $\omega_{1}, \ldots, \omega_{n-1}$ be the dual basis of $(1,0)$-forms that satisfy $\left\langle\omega_{j}, L_{k}\right\rangle=\delta_{j k}$. Then $\bar{L}_{1}, \ldots, \bar{L}_{n-1}$ is a local orthonormal basis for the $(0,1)$-vector fields with dual basis $\bar{\omega}_{1}, \ldots, \bar{\omega}_{n-1}$ in $U$. Also, the tangent bundle $T(U)$ is spanned by $L_{1}, \ldots, L_{n-1}, \bar{L}_{1}, \ldots, \bar{L}_{n-1}$, and an additional vector field $T$ taken to be purely imaginary (so $\bar{T}=-T$ ).

Since $M$ is orientable, there exists a global, purely imaginary 1-form $\gamma$ on $M$ that annihilates $T^{1,0}(M) \oplus T^{0,1}(M)$ and is normalized so that $\langle\gamma, T\rangle=-1$.

Definition 1.1. The Levi form at a point $x \in M$ is the Hermitian form given by $-\left\langle\gamma_{x},\left[L, \bar{L}^{\prime}\right]\right\rangle$ where $L, L^{\prime} \in T_{x}^{1,0}(U)$, and $U$ is a neighborhood of $x \in M$.

We remark that $-\left\langle\gamma_{x},\left[L, \bar{L}^{\prime}\right]\right\rangle=\left\langle d \gamma, L \wedge \bar{L}^{\prime}\right\rangle$ since $\gamma$ annihilates $T^{1,0}(M) \oplus$ $T^{0,1}(M)$.

Recall that $M$ is pseudoconvex if, for some orientation of $\gamma$, the Levi form is positive semi-definite at all $x \in M$ and strictly pseudoconvex if, for some orientation of $\gamma$, the Levi form is positive definite at all $x \in M$.

When $q$ is fixed, strict pseudoconvexity is not necessary to prove $1 / 2$ estimates for the $\bar{\partial}$-Neumann operator. Instead, the optimal condition is $Z(q)$ (see, e.g., [FK72,CS01]). $M$ is said to satisfy $Z(q), 1 \leq q \leq n-1$, if the Levi form associated with $M$ has at least $n-q$ positive eigenvalues or at least $q+1$ negative eigenvalues. $M$ is said to satisfy $Y(q), 1 \leq q \leq n-1$, if $M$ satisfies $Z(q)$ and $Z(n-1-q)$. The necessity of the symmetric requirements for $\bar{\partial}_{b}$ at levels $q$ and $n-1-q$ stems from the duality between $(0, q)$-forms and $(0, n-1-q)$-forms (see [FK72] or [RS08] for details).

Our definition of weak $Z(q)$ follows $\underline{\mathrm{HR}}$.

Definition 1.2. Let $M \subset \mathbb{C}^{n}$ be a smooth, compact, orientable CR manifold of hypersurface type. We say that $M$ satisfies weak $Z(q)$ if there exists a real bivector $\Upsilon \in T^{1,1}(M)$ that satisfies:

(i) $|\omega|^{2} \geq(i \omega \wedge \bar{\omega})(\Upsilon) \geq 0$, for all $\omega \in \Lambda^{1,0}(M)$;

(ii) $\mu_{1}+\cdots+\mu_{q}-d \gamma(\Upsilon) \geq 0$, where $\mu_{1}, \ldots, \mu_{n-1}$ are the eigenvalues of the Levi form in increasing order; 
(iii) $\inf _{M}\{|q-\operatorname{Tr}(\Upsilon)|\}>0$.

As above, $M$ satisfies weak $Y(q)$ at $x$ if $M$ satisfies weak $Z(q)$ at $x$ and weak $Z(n-1-q)$ at $x$.

Remark 1.3. In local coordinates, $\Upsilon$ may be identified with an $(n-1) \times(n-1)$ Hermitian matrix $\left(a_{j k}\right)$ via $\Upsilon=\sum_{j, k} i a_{j k} \bar{L}_{k} \wedge L_{j}$.

1.2. Weak $Z(q)$ and the basic estimate. In this part, we provide motivation and commentary on the weak $Z(q)$ condition.

Let $\Omega \subset \mathbb{C}^{n}$ be a smooth, bounded domain. Let $\mathcal{I}_{q}=\left\{J=\left(j_{1}, \ldots, j_{q}\right): 1 \leq\right.$ $\left.j_{1}<\cdots<j_{q} \leq n\right\}$. For $f, g \in L_{0, q}^{2}(\Omega)$, define

$$
(f, g)_{t}=\sum_{J \in \mathcal{I}_{q}} \int_{\Omega} f_{J}(z) \overline{g_{J}(z)} e^{-t|z|^{2}} d V(z)
$$

and $\|f\|_{t, L^{2}(\Omega)}^{2}=(f, f)_{t}$. Let $\bar{\partial}_{t}^{*}$ be the $L^{2}$ adjoint of $\bar{\partial}$ with respect to the $(\cdot, \cdot)_{t}$ sesquilinear product. Let $\mathrm{b} \Omega$ be the boundary of $\Omega, \rho$ a defining function for $\Omega$ with $|\nabla \rho|=1$ on $\mathrm{b} \Omega$, and $d \sigma$ be the induced surface area measure on $\mathrm{b} \Omega$. A classical version of the basic identity (or Kohn-Morrey formula) is

$$
\begin{aligned}
\|\bar{\partial} f\|_{t, L^{2}(\Omega)}^{2}+\left\|\bar{\partial}_{t}^{*} f\right\|_{t, L^{2}(\Omega)}^{2}= & \sum_{J \in \mathcal{I}_{q}} \sum_{j=1}^{n} \int_{\Omega}\left|\frac{\partial f_{J}}{\partial \bar{z}_{j}}\right|^{2} e^{-t|z|^{2}} d V+q t\|f\|_{t, L^{2}(\Omega)}^{2} \\
& +\sum_{K \in \mathcal{I}_{q-1}} \sum_{j, k=1}^{n} \int_{\mathrm{b} \Omega} \frac{\partial^{2} \rho(z)}{\partial z_{j} \partial \bar{z}_{k}} u_{j K} \overline{u_{k K}} e^{-t|z|^{2}} d \sigma(z) .
\end{aligned}
$$

See [Str10, Proposition 2.4] for a proof. A closed range estimate for $\bar{\partial}$ follows from this identity if the boundary integral is positive and $t>0$. If $\Omega$ is pseudoconvex (or at least the sum of any $q$ eigenvalues of the Levi form is nonnegative), then the boundary integral will be positive.

When $\Omega$ is not pseudoconvex, then (1) is not necessarily a useful equality. For example, if $\Omega$ is an annular region between two pseudoconvex domains, i.e., $\Omega=$ $\Omega_{1} \backslash \overline{\Omega_{2}}$ where $\Omega_{1} \supset \Omega_{2}$ and both domains are pseudoconvex, then near b $\Omega_{2}$ it is helpful to integrate the $\left(\frac{\partial f_{J}}{\partial \bar{z}_{j}}, \frac{\partial f_{J}}{\partial \bar{z}_{j}}\right)_{t}$ terms by parts. If we set $L_{j}^{t}=\frac{\partial}{\partial z_{j}}-t \bar{z}_{j}=$ $e^{t|z|^{2}} \frac{\partial}{\partial z_{j}} e^{-t|z|^{2}}$ and $\rho_{j \bar{k}}=\frac{\partial^{2} \rho}{\partial z \partial \bar{z}_{k}}$,

$$
\begin{aligned}
& \text { (2) }\|\bar{\partial} f\|_{t, L^{2}(\Omega)}^{2}+\left\|\bar{\partial}_{t}^{*} f\right\|_{t, L^{2}(\Omega)}^{2}=\sum_{J \in \mathcal{I}_{q}} \sum_{j, k=1}^{n}\left\|L_{j}^{t} f_{J}\right\|_{t, L^{2}(\Omega)}^{2}-t(n-q)\|f\|_{t, L^{2}(\Omega)}^{2} \\
& +\sum_{I \in \mathcal{I}_{q-1}} \sum_{j, k=1}^{n} \int_{\mathrm{b} \Omega} \rho_{j \bar{k}} f_{j I} \overline{f_{k I}} e^{-t|z|^{2}} d \sigma-\sum_{J \in \mathcal{I}_{q}} \int_{\mathrm{b} \Omega} \operatorname{Tr}\left(\rho_{j} \bar{k}\right)\left|f_{J}\right|^{2} e^{-t|z|^{2}} d \sigma+O\left(\|f\|_{t, L^{2}(\Omega)}^{2}\right) .
\end{aligned}
$$

Equation (2) works where $b \Omega$ is pseudoconcave since the eigenvalues of the Levi form are nonpositive. We also need $t<0$ for a closed range estimate.

The $(q-1)$-pseudoconcave property stems from the idea that we do not have to integrate by parts all of the $(0,1)$ vector fields. For example, if we arranged the eigenvalues of the Levi form in increasing order and had a coordinate system where the $j$ th coordinate was associated with the $j$ th eigenvalue of the Levi from (e.g., if the Levi form was diagonalizable), then an effective identity would be a combination of (1) and (2). Certain $(1,0)$ and $(0,1)$ vector fields appear, and we 
do not subtract the full trace of the Levi form. In fact, we get a basic identity of the form

$$
\begin{aligned}
& \|\bar{\partial} f\|_{t, L^{2}(\Omega)}^{2}+\left\|\bar{\partial}_{t}^{*} f\right\|_{t, L^{2}(\Omega)}^{2} \\
= & \sum_{J \in \mathcal{I}_{q}} \sum_{k=m+1}^{n}\left\|\frac{\partial f_{J}}{\partial \bar{z}_{k}}\right\|_{t, L^{2}(\Omega)}^{2}+\sum_{J \in \mathcal{I}_{q}} \sum_{j=1}^{m}\left\|L_{j} f_{J}\right\|_{t, L^{2}(\Omega)}^{2}+t(q-m)\|f\|_{t, L^{2}(\Omega)}^{2} \\
+ & \int_{\mathrm{b} \Omega}\left[\sum_{I \in \mathcal{I}_{q-1}} \sum_{j, k=1}^{n} \rho_{j \bar{k}} f_{j I} \overline{f_{k I}} e^{-t|z|^{2}} d \sigma-\sum_{j=1}^{m} \rho_{j \bar{j}}|f|^{2} e^{-t|z|^{2}}\right] d \sigma+O\left(\|f\|_{t, L^{2}(\Omega)}^{2}\right) .
\end{aligned}
$$

The sign of $t$ depends on whether $m>q$ or $m<q$, and this depends on how many eigenvalues of the Levi form are negative. The only value that $m$ is not allowed to take is $m=q$. Zampieri's $(q-1)$-pseudoconvexity is a condition that requires a vector bundle of dimension $m$ so that the boundary integral in (3) is always a positive term and $m<q$. In [HR11], Harrington and Raich permitted the case $m>q$, which is useful when dealing with annular regions.

In [HR], Harrington and Raich introduced a matrix $\Upsilon$ (relative to a choice of basis for $T^{p, q}(M)$; see Remark 1.3) that governs the integration by parts. In the pseudoconvex convex case, $\Upsilon$ is the 0 matrix (no integration by parts needed). In the pseudoconcave case, $\Upsilon=I$, the identity matrix, since every $(0,1)$ vector field needs to be integrated by parts. In the $(q-1)$-pseudoconcave case (or weak $Z(q)$ case with the definition from [HR11],$\Upsilon$ is diagonal and has the form

$$
\Upsilon=\left(\begin{array}{cc}
I_{m} & 0 \\
0 & 0
\end{array}\right)
$$

where $I_{m}$ is the $m \times m$ identity matrix. In looking at the basic identities, (1), (2), and (3), Harrington and Raich observed three items in trying to form the matrix $\Upsilon$ :

i. We need $0 \leq \Upsilon \leq I$ or the sum of the $(0,1)$ and $(1,0)$ vector fields may not be positive.

ii. $\Upsilon$ must be chosen so that the boundary integral is positive.

iii. $\Upsilon$ cannot cause the $L^{2}$ norm of $f$ that is multiplied by $t$ to vanish. This is the $t(q-m)\|f\|_{t, L^{2}(\Omega)}^{2}$ term in (3).

Given the requirements on $\Upsilon=\left(\Upsilon^{j \bar{k}}\right)$, they formulated the weak $Z(q)$ condition for domains in a Stein manifold. In the case of an embedded CR manifold of hypersurface type, this definition becomes Definition [1.2. The basic identity for a smooth, bounded pseudoconvex domain $\Omega \subset \mathbb{C}^{n}$ is then

$$
\begin{aligned}
& \|\bar{\partial} f\|_{t, L^{2}(\Omega)}^{2}+\left\|\bar{\partial}_{t}^{*} f\right\|_{t, L^{2}(\Omega)}^{2}=\sum_{J \in \mathcal{I}_{q}} \sum_{j, k=1}^{n}\left(\left(\delta_{j k}-\Upsilon^{\bar{k} j}\right) \frac{\partial f_{J}}{\partial \bar{z}_{k}}, \frac{\partial f_{J}}{\partial \bar{z}_{j}}\right)_{t}+\sum_{J \in \mathcal{I}_{q}} \sum_{j, k=1}^{n}\left(\Upsilon^{\bar{k} j} L_{j}^{t} f_{J}, L_{k}^{t} f_{J}\right)_{t} \\
& +\sum_{I \in \mathcal{I}_{q-1}} \sum_{j, k=1}^{n} \int_{\mathrm{b} \Omega} \rho_{j \bar{k}} f_{j I} \overline{f_{k I}} e^{-t|z|^{2}} d \sigma-\sum_{j, k=1}^{n} \int_{b \Omega} \Upsilon^{\bar{k} j} \rho_{j \bar{k}}|f|^{2} e^{-t|z|^{2}} d \sigma \\
& +2 \operatorname{Re}\left\{\sum_{J \in \mathcal{I}_{q}} \sum_{j, k, \ell=1}^{n}\left(\frac{\partial \Upsilon^{\bar{k} j} j}{\partial \bar{z}_{k}} \Upsilon^{\bar{j} \ell} L_{\ell}^{t} f_{J}, f_{J}\right)_{t}-\sum_{J \in \mathcal{I}_{q}} \sum_{j, k, \ell=1}^{n}\left(\frac{\partial \Upsilon^{\bar{k} j}}{\partial z_{j}}\left(\delta_{k \ell}-\Upsilon^{\bar{\ell} k}\right) \frac{\partial f_{J}}{\partial \bar{z}_{\ell}}, f_{J}\right)_{t}\right\} \\
& +\sum_{J \in \mathcal{I}_{q}} t\left((q-\operatorname{Tr}(\Upsilon)) f_{J}, f_{J}\right)_{t}+O\left(\|f\|_{t, L^{2}(\Omega)}^{2}\right),
\end{aligned}
$$

where $O\left(\|f\|_{t, L^{2}(\Omega)}^{2}\right) \leq C\left(\|\Upsilon\|_{C^{2}(\bar{\Omega})}+\|\Upsilon\|_{C^{2}(\bar{\Omega})}^{2}\right)\|f\|_{t, L^{2}(\Omega)}$. This identity includes (11), (2), and (3) as special cases, as discussed above. 
1.3. Norms. We follow the notation from [HR11, Section 3]. We set

$$
(\varphi, \phi)_{t}=\int_{M} \phi \bar{\varphi} e^{-t|z|^{2}} d \sigma .
$$

In particular, $t=0$ is the standard, unweighted $L^{2}$ inner product and has norm $\|\varphi\|_{L^{2}(M)}^{2}=(\varphi, \varphi)_{0}$.

We follow the setup for the microlocal analysis in Rai10, HR11. Since $M$ is compact, there exists a finite cover $\left\{U_{\nu}\right\}_{\nu}$ so each $U_{\nu}$ has a special boundary system and can be parameterized by a hypersurface in $\mathbb{C}^{n}\left(U_{\nu}\right.$ may be shrunk as necessary). To set up the microlocal analysis, we need to define the appropriate pseudodifferential operators on each $U_{\nu}$. Let $\xi=\left(\xi_{1}, \ldots, \xi_{2 n-2}, \xi_{2 n-1}\right)=\left(\xi^{\prime}, \xi_{2 n-1}\right)$ be the coordinates in Fourier space so that $\xi^{\prime}$ is dual to the part of $T(M)$ in the maximal complex subspace (i.e., $\left.T^{1,0}(M) \oplus T^{0,1}(M)\right)$ and $\xi_{2 n-1}$ is dual to the totally real part of $T(M)$, i.e., the "bad" direction $T$. Define

$$
\begin{aligned}
& \mathcal{C}^{+}=\left\{\xi: \xi_{2 n-1} \geq \frac{1}{2}\left|\xi^{\prime}\right| \text { and }|\xi| \geq 1\right\} \\
& \mathcal{C}^{-}=\left\{\xi:-\xi \in \mathcal{C}^{+}\right\} \\
& \mathcal{C}^{0}=\left\{\xi:-\frac{3}{4}\left|\xi^{\prime}\right| \leq \xi_{2 n-1} \leq \frac{3}{4}\left|\xi^{\prime}\right|\right\} \cup\{\xi:|\xi| \leq 1\} .
\end{aligned}
$$

Note that $\mathcal{C}^{+}$and $\mathcal{C}^{-}$are disjoint, but both intersect $\mathcal{C}^{0}$ nontrivially. Next, we define smooth functions on $\left\{|\xi|:|\xi|^{2}=1\right\}$. Let

$$
\begin{aligned}
& \psi^{+}(\xi)=1 \text { when } \xi_{2 n-1} \geq \frac{3}{4}\left|\xi^{\prime}\right| \text { and } \operatorname{supp} \psi^{+} \subset\left\{\xi: \xi_{2 n-1} \geq \frac{1}{2}\left|\xi^{\prime}\right|\right\} \\
& \psi^{-}(\xi)=\psi^{+}(-\xi) \\
& \psi^{0}(\xi) \text { satisfies } \psi^{0}(\xi)^{2}=1-\psi^{+}(\xi)^{2}-\psi^{-}(\xi)^{2} .
\end{aligned}
$$

Extend $\psi^{+}, \psi^{-}$, and $\psi^{0}$ homogeneously outside of the unit ball; i.e., if $|\xi| \geq 1$, then

$$
\psi^{+}(\xi)=\psi^{+}(\xi /|\xi|), \psi^{-}(\xi)=\psi^{-}(\xi /|\xi|) \text {, and } \psi^{0}(\xi)=\psi^{0}(\xi /|\xi|) .
$$

Also, extend $\psi^{+}, \psi^{-}$, and $\psi^{0}$ smoothly inside the unit ball so that $\left(\psi^{+}\right)^{2}+\left(\psi^{-}\right)^{2}+$ $\left(\psi^{0}\right)^{2}=1$. Finally, there exists a large constant $A>0$ that depends on $M$ (which allows the weighted Sobolev theory to hold and whose existence is proven in Rai10. HR11) when we define, for any $t>0$,

$$
\psi_{t}^{+}(\xi)=\psi^{+}(\xi /(t A)), \psi_{t}^{-}(\xi)=\psi^{-}(\xi /(t A)), \text { and } \psi_{t}^{0}(\xi)=\psi^{0}(\xi /(t A)) .
$$

Next, let $\Psi_{t}^{+}, \Psi_{t}^{-}$, and $\Psi_{t}^{0}$ be the pseudodifferential operators of order zero with symbols $\psi_{t}^{+}, \psi_{t}^{-}$, and $\psi_{t}^{0}$, respectively. The equality $\left(\psi_{t}^{+}\right)^{2}+\left(\psi_{t}^{-}\right)^{2}+\left(\psi_{t}^{0}\right)^{2}=1$ implies that

$$
\left(\Psi_{t}^{+}\right)^{*} \Psi_{t}^{+}+\left(\Psi_{t}^{0}\right)^{*} \Psi_{t}^{0}+\left(\Psi_{t}^{-}\right)^{*} \Psi_{t}^{-}=I d .
$$

We will also have use for pseudodifferential operators that "dominate" a given pseudodifferential operator. Let $\psi$ be a cut-off function and $\tilde{\psi}$ be another cut-off function so that $\left.\tilde{\psi}\right|_{\operatorname{supp} \psi} \equiv 1$. If $\Psi$ and $\tilde{\Psi}$ are pseudodifferential operators with symbols $\psi$ and $\tilde{\psi}$, respectively, then we say that $\tilde{\Psi}$ dominates $\Psi$.

For each $U_{\nu}$, we can define $\Psi_{t}^{+}, \Psi_{t}^{-}$, and $\Psi_{t}^{0}$ to act on functions or forms supported in $U_{\nu}$, so let $\Psi_{t, \nu}^{+}, \Psi_{t, \nu}^{-}$, and $\Psi_{t, \nu}^{0}$ be the pseudodifferential operators of order zero defined on $U_{\nu}$, and let $\mathcal{C}_{\nu}^{+}, \mathcal{C}_{\nu}^{-}$, and $\mathcal{C}_{\nu}^{0}$ be the regions of $\xi$-space dual to $U_{\nu}$ on 
which the symbol of each of those pseudodifferential operators is supported. Then it follows that

$$
\left(\Psi_{t, \nu}^{+}\right)^{*} \Psi_{t, \nu}^{+}+\left(\Psi_{t, \nu}^{0}\right)^{*} \Psi_{t, \nu}^{0}+\left(\Psi_{t, \nu}^{-}\right)^{*} \Psi_{t, \nu}^{-}=I d
$$

Let $\left\{\zeta_{\nu}\right\}$ be a partition of unity subordinate to the covering $\left\{U_{\nu}\right\}$ satisfying $\sum_{\nu} \zeta_{\nu}^{2}=1$. Also, for each $\nu$, let $\tilde{\zeta}_{\nu}$ be a cutoff function that dominates $\zeta_{\nu}$ so that $\operatorname{supp} \tilde{\zeta}_{\nu} \subset U_{\nu}$. We define

$$
\begin{aligned}
\langle\phi, \varphi\rangle_{t}=\sum_{\nu}\left[\left(\tilde{\zeta}_{\nu} \Psi_{\nu, t}^{+} \zeta_{\nu} \phi^{\nu}, \tilde{\zeta}_{\nu} \Psi_{\nu, t}^{+} \zeta_{\nu} \varphi^{\nu}\right)_{\lambda_{+} t}\right. & \\
& \left.\quad+\left(\tilde{\zeta}_{\nu} \Psi_{\nu, t}^{0} \zeta_{\nu} \phi^{\nu}, \tilde{\zeta}_{\nu} \Psi_{\nu, t}^{0} \zeta_{\nu} \varphi^{\nu}\right)_{0}+\left(\tilde{\zeta}_{\nu} \Psi_{\nu, t}^{-} \zeta_{\nu} \phi^{\nu}, \tilde{\zeta}_{\nu} \Psi_{\nu, t}^{-} \zeta_{\nu} \varphi^{\nu}\right)_{\lambda_{-} t}\right]
\end{aligned}
$$

where

$$
\lambda_{+}= \begin{cases}1 & \text { if } \operatorname{Tr} \Upsilon<q, \\ -1 & \text { if } \operatorname{Tr} \Upsilon>q\end{cases}
$$

and

$$
\lambda_{-}= \begin{cases}-1 & \text { if } \operatorname{Tr} \Upsilon<n-1-q, \\ 1 & \text { if } \operatorname{Tr} \Upsilon>n-1-q .\end{cases}
$$

Set

$$
\|\varphi\|_{t}^{2}=\langle\varphi, \varphi\rangle_{t}
$$

Let $\Lambda^{s}$ be the pseudodifferential operator with symbol $\left(1+|\xi|^{2}\right)^{s / 2}$. We set the Sobolev $s$-norm on $W^{s}(M)$ to be

$$
\|\varphi\|_{W^{s}(M)}^{2}=\sum_{\nu}\left\|\tilde{\zeta}_{\nu} \Lambda^{s} \zeta_{\nu} \varphi^{\nu}\right\|_{L^{2}(M)}^{2}
$$

It is shown in Nic06, Rai10, that there exist constants $c_{t}, C_{t}>0$ so that

$$
c_{t}\|\varphi\|_{L^{2}(M)}^{2} \leq\|\varphi\|_{t}^{2} \leq C_{t}\|\varphi\|_{L^{2}(M)}^{2}
$$

and an invertible pseudodifferential operator of order $0, F_{t}$, so that

$$
\langle\varphi, \phi\rangle_{t}=\left(\varphi, F_{t} \phi\right)_{0} .
$$

1.4. $L^{2}$ theory for $\bar{\partial}_{b}$. In [HR11, Harrington and Raich established Kohn's weighted theory for $\bar{\partial}_{b}$. In particular, let $\bar{\partial}_{b, t}^{*}$ be the $L^{2}$-adjoint of $\bar{\partial}_{b}$ in $\langle\cdot, \cdot\rangle_{t}$, $\square_{b, t}=\bar{\partial}_{b} \bar{\partial}_{b, t}^{*}+\bar{\partial}_{b, t}^{*} \bar{\partial}_{b}, H_{q, t}$ the projection of $L_{0, q}^{2}\left(M, e^{-t|z|^{2}}\right)$ onto ker $\bar{\partial}_{b} \cap \operatorname{ker} \bar{\partial}_{b, t}^{*}$, and $G_{q, t}$ be the relative inverse to $\square_{b, t}$, that is, the inverse on the orthogonal complement of $\operatorname{ker} \square_{b, t}$. When $t=0$, we suppress the subscript. We also know that $\bar{\partial}_{b}^{*}-\bar{\partial}_{b, t}^{*}$ is an operator of order 0 from [Rai10, Lemma 3.7].

We have the Hodge decomposition

$$
I=\bar{\partial}_{b} \bar{\partial}_{b}^{*} G_{q}+\bar{\partial}_{b}^{*} \bar{\partial}_{b} G_{q}+H_{q}
$$

and a similar Hodge decomposition for the weighted operators. Let $S_{q}: L_{0, q}^{2}(M) \rightarrow$ ker $\bar{\partial}_{b}$ be the Szegö projection. Since $S_{q}$ is self-adjoint, it follows that $\operatorname{ker} S_{q}=$ $\left(\text { Range } S_{q}\right)^{\perp}$. It is also easily checked that $S_{q} \bar{\partial}_{b}^{*}=0$, so

$$
S_{q}=\bar{\partial}_{b} \bar{\partial}_{b}^{*} G_{q}+H_{q},
$$

and therefore Kohn's formula

$$
S_{q}=I-\bar{\partial}_{b}^{*} \bar{\partial}_{b} G_{q}
$$


holds. Since we do not know that $G_{q-1}$ exists as a continuous operator on $L_{0, q-1}^{2}(M)$ (and hence cannot commute $G_{q-1}$ with $\bar{\partial}_{b}$ ), we define

$$
S_{q-1}=I-\bar{\partial}_{b}^{*} G_{q} \bar{\partial}_{b}
$$

Then $S_{q-1}$ is a self-adjoint projection and hence is still an orthogonal projection. We will continue to call $S_{q-1}$ a Szegö projection because if we had a Hodge theory for $L_{0, q-1}^{2}(M)$, then $S_{q-1}$ would agree with the Szegö projection as defined above. We also set

$$
S_{q+1}^{\prime}=\bar{\partial}_{b} G_{q} \bar{\partial}_{b}^{*}
$$

The orthogonal projection $S_{q+1}^{\prime}$ is not generically the Szegö projection because it annihilates harmonic forms.

Every formula in this section has a weighted analog.

\section{Statements of the main Results}

In what follows, we reserve $t \geq 0$ for the weight $\lambda_{t}(z)=e^{-t|z|^{2}}$ and $s \geq 0$ for Sobolev norms of order $s$ (defined below).

\subsection{CR manifolds of hypersurface type.}

Theorem 2.1. Let $M$ be a smooth, compact, embedded, $C R$ manifold of hypersurface type that satisfies weak $Y(q)$ for some $1 \leq q \leq n-2$. Let $s \geq 0$. If $G_{q}$ is a continuous operator on $W_{0, q}^{s+2}(M)$, then there exists a constant $C_{r}$ so that, for every $u \in C^{\infty}(M)$,

$$
\left\|S_{q-1} u\right\|_{W^{r}(M)}+\left\|S_{q} u\right\|_{W^{r}(M)}+\left\|S_{q+1}^{\prime} u\right\|_{W^{r}(M)} \leq C_{r}\left\|G_{q} u\right\|_{W^{r}(M)}
$$

for $0 \leq r \leq s$.

If $\bar{S}_{q-1}, S_{q}$, and $S_{q+1}^{\prime}$ are continuous operators on $W_{0, q-1}^{s}(M), W_{0, q}^{s}(M)$, and $W_{0, q+1}^{s}(M)$, respectively, then $G_{q}$ is a continuous operator on $W_{0, q}^{s}(M)$ and there exists a constant $C_{s}$ so that for every $u \in C^{\infty}(M)$,

$$
\left\|G_{q} u\right\|_{W^{s}(M)} \leq C_{s}\left(\left\|S_{q-1} u\right\|_{W^{s}(M)}+\left\|S_{q} u\right\|_{W^{s}(M)}+\left\|S_{q+1}^{\prime} u\right\|_{W^{s}(M)}\right) .
$$

Corollary 2.2. Let $M$ be a smooth, compact, embedded, CR manifold of hypersurface type that satisfies weak $Y(q)$ for some $1 \leq q \leq n-2$. Then $G_{q}$ is exactly regular if and only if $S_{q-1}, S_{q}$, and $S_{q+1}^{\prime}$ are exactly regular.

Proposition 2.3. Let $M \subset \mathbb{C}^{N}$ be a smooth, compact, embedded, CR manifold of hypersurface type that satisfies weak $Y(q)$. Let $k \in \mathbb{Z}$ be a positive integer. If $u$ and $G_{q} u$ are both in $W_{0, q}^{k+2}(M)$ and $u \perp \mathcal{H}_{q}$, then there exists a constant $C>0$ so that

$$
\begin{array}{r}
\left\|\bar{\partial}_{b}^{*} \bar{\partial}_{b} G_{q} u\right\|_{W^{k}(M)}+\left\|\bar{\partial}_{b} \bar{\partial}_{b}^{*} G_{q} u\right\|_{W^{k}(M)}+\left\|\bar{\partial}_{b} G_{q} u\right\|_{W^{k}(M)}+\left\|\bar{\partial}_{b}^{*} G_{q} u\right\|_{W^{k}(M)} \\
\leq C\left(\left\|G_{q} u\right\|_{W^{k}(M)}+\|u\|_{W^{k}(M)}\right) .
\end{array}
$$

Proposition 2.3 should be compared with [Str10, Lemma 3.2]. 
2.2. Smooth, bounded domains in a Stein manifold. We have a similar group of results for smooth, bounded domains in a Stein manifold.

Theorem 2.4. Let $M$ be a Stein manifold and $\Omega \subset M$ a smooth, bounded domain that satisfies weak $Z(q)$ for some $1 \leq q \leq n-1$. Let $s \geq 0$. If $N_{q}$ is a continuous operator on $W_{0, q}^{s+2}(\Omega)$, then there exists a constant $C_{r}$ so that

$$
\left\|P_{q-1} u\right\|_{W^{r}(\Omega)}+\left\|P_{q} u\right\|_{W^{r}(\Omega)}+\left\|P_{q+1}^{\prime} u\right\|_{W^{r}(\Omega)} \leq C_{r}\left\|N_{q} u\right\|_{W^{r}(\Omega)}
$$

for all $u \in C^{\infty}(\Omega)$ and for $0 \leq r \leq s$.

If $P_{q-1}, P_{q}$, and $P_{q+1}^{\prime}$ are continuous operators on $W_{0, q-1}^{s}(M), W_{0, q}^{s}(M)$, and $W_{0, q+1}^{s}(M)$, respectively, then $N_{q}$ is a continuous operator on $W_{0, q}^{s}(M)$ and there exists a constant $C_{s}$ so that

$$
\left\|N_{q}\right\|_{W^{s}(\Omega)} \leq C_{s}\left(\left\|P_{q-1}\right\|_{W^{s}(\Omega)}+\left\|P_{q}\right\|_{W^{s}(\Omega)}+\left\|P_{q+1}^{\prime}\right\|_{W^{s}(\Omega)}\right) .
$$

Corollary 2.5. Let $M$ be a Stein manifold and $\Omega \subset M$ a smooth, bounded domain that satisfies weak $Z(q)$ for some $1 \leq q \leq n-1$. Then $N_{q}$ is exactly regular if and only if $P_{q-1}, P_{q}$, and $P_{q+1}^{\prime}$ are exactly regular.

Proposition 2.6. Let $M$ be a Stein manifold and $\Omega \subset M$ a smooth, bounded domain that satisfies weak $Z(q)$ for some $1 \leq q \leq n-1$. Let $k \in \mathbb{Z}$ be a positive integer. If $u$ and $N_{q} u$ are both in $W_{0, q}^{k+2}(M)$ and $u \perp \mathcal{H}_{q}$, then there exists a constant $C>0$ so that

$$
\begin{array}{r}
\left\|\bar{\partial}^{*} \bar{\partial} N_{q} u\right\|_{W^{k}(\Omega)}+\left\|\bar{\partial} \bar{\partial}^{*} N_{q} u\right\|_{W^{k}(\Omega)}+\left\|\bar{\partial} N_{q} u\right\|_{W^{k}(\Omega)}+\left\|\bar{\partial}^{*} N_{q} u\right\|_{W^{k}(\Omega)} \\
\leq C\left(\left\|N_{q} u\right\|_{W^{k}(\Omega)}+\|u\|_{W^{k}(\Omega)}\right) .
\end{array}
$$

In summary, we have generalized the approach of [BS90] in several ways.

First, we deal with the boundary analogue, that is, with the complex Green operator and the Szegö projection. Second, we do not require pseudoconvexity and instead focus on obtaining results for a fixed $q, 1 \leq q \leq n$. Third, we reduce the regularity hypotheses in the relationship between the Szegö (resp., Bergman) projection and the complex Green (resp., $\bar{\partial}$-Neumann) operator. Finally, we wanted to establish that the regularity arguments are quite general and require only an appropriate weighted Sobolev theory and Hodge-*decomposition. We provide two examples where the first and third authors have established the necessary ingredients.

\section{Proofs of Theorem 2.1 And Proposition 2.3}

In [HR11, Harrington and Raich discussed how the regularity of $G_{q, t} \bar{\partial}_{b}$ and $G_{q, t} \bar{\partial}_{b, t}^{*}$ follows from the regularity of $G_{q, t}$. We provide a proof of this fact for completeness.

Proposition 3.1. Let $M$ be a smooth CR manifold of hypersurface type that satisfies the hypotheses of Theorem 2.1. For each $s \geq 0$, there exists $T_{s}$ so that if $t \geq T_{s}$ then $G_{q, t} \bar{\partial}_{b}: W_{0, q-1}^{s}(M) \rightarrow W_{0, q}^{s}(M)$ and $G_{q, t} \bar{\partial}_{b, t}^{*}: W_{0, q+1}^{s}(M) \rightarrow W_{0, q}^{s}(M)$ continuously.

Proof. We show that $G_{q, t} \bar{\partial}_{b, t}^{*}: W_{0, q+1}^{s}(\Omega) \rightarrow W_{0, q}^{s}(\Omega)$ and $G_{q, t} \bar{\partial}_{b}: W_{0, q-1}^{s}(M) \rightarrow$ $W_{0, q}^{s}(M)$ continuously. The cases $s=0$ and $s=1$ are proven in [HR, Theorem 1.2] 
(see also [HR, Theorem 4.3]. We can adapt Harrington and Raich's argument from [HR] for larger $s$. Observe that

$$
\bar{\partial}_{b} \Lambda^{s} G_{q, t} f=\left[\bar{\partial}_{b}, \Lambda^{s}\right] G_{q, t} f+\Lambda^{s} \bar{\partial}_{b} G_{q, t} f
$$

and

$$
\bar{\partial}_{b, t}^{*} \Lambda^{s} G_{q, t} f=\left[\bar{\partial}_{b, t}^{*}, \Lambda^{s}\right] G_{q, t} f+\Lambda^{s} \bar{\partial}_{b, t}^{*} G_{q, t} f .
$$

Implicit in HR11] is the fact that if $\epsilon>0$, then for $t$ large enough we have

$$
\left\|\Lambda^{s} G_{q, t} f \mid\right\|_{t}^{2} \leq \epsilon\left(\left\|\bar{\partial}_{b} \Lambda^{s} G_{q, t} f\right\|_{t}^{2}+\left\|\bar{\partial}_{b, t}^{*} \Lambda^{s} G_{q, t} f\right\|_{t}^{2}\right)+C_{t}\left\|\Lambda^{s-1} G_{q, t} f\right\|_{t}^{2} .
$$

Since $f$ has smooth coefficients, by choosing $t$ larger (if necessary), we can use a small constant/large constant argument and estimate

$$
\left\|\mid \Lambda^{s} G_{q, t} f\right\|_{t}^{2} \leq \epsilon\left(\left\|\Lambda^{s} \bar{\partial}_{b} G_{q, t} f\right\|_{t}^{2}+\left\|\Lambda^{s} \bar{\partial}_{b, t}^{*} G_{q, t} f\right\|_{t}^{2}\right)+C_{t}\left\|\Lambda^{s-1} G_{q, t} f\right\|_{t}^{2} .
$$

Next, suppose that $f=\bar{\partial}_{b, t}^{*} g$ for a $(0, q+1)$-form with smooth coefficients. Using induction in $s$ to control $\left\|\mid \Lambda^{s-1} G_{q, t} \bar{\partial}_{b, t}^{*} g\right\|_{t}$, we have

$$
\left\|\left|\Lambda^{s} G_{q, t} \bar{\partial}_{b, t}^{*} g\right|\right\|_{t}^{2} \leq \epsilon\left\|\Lambda^{s} \bar{\partial}_{b} G_{q, t} \bar{\partial}_{b, t}^{*} g\right\|_{t}^{2}+C_{t}\left\|\Lambda^{s-1} g\right\| \|_{t}^{2} .
$$

We now handle the term $\left\|\mid \Lambda^{s} \bar{\partial}_{b} G_{q, t} \bar{\partial}_{b, t}^{*} g\right\|_{t}^{2}$. Observe that $\left[\bar{\partial}_{b, t}^{*}, \Lambda^{s}\right]=O\left(\Lambda^{s}\right)+$ $t O\left(\Lambda^{s-1}\right)$. We adopt the convention that the constant implicit in the error terms is independent of $t$, and we use $C_{t}$ to represent constants depending on $t$. We estimate

$$
\begin{aligned}
& \left\|\mid \Lambda^{s} \bar{\partial}_{b} G_{q, t} \bar{\partial}_{b, t}^{*} g\right\|_{t}^{2} \\
& =\left\langle\Lambda^{s} G_{q, t} \bar{\partial}_{b, t}^{*} g, \bar{\partial}_{b, t}^{*} \Lambda^{s} \bar{\partial}_{b} G_{q, t} \bar{\partial}_{b, t}^{*} g\right\rangle_{t}+\left\langle\left[\Lambda^{s}, \bar{\partial}_{b}\right] G_{q, t} \bar{\partial}_{b, t}^{*} g, \Lambda^{s} \bar{\partial}_{b} G_{q, t} \bar{\partial}_{b, t}^{*} g\right\rangle_{t} \\
& \leq\left|\left\langle\Lambda^{s} G_{q, t} \bar{\partial}_{b, t}^{*} g, \Lambda^{s} \bar{\partial}_{b, t}^{*} \bar{\partial}_{b} G_{q, t} \bar{\partial}_{b, t}^{*} g\right\rangle_{t}\right| \\
& \quad+O\left(\left\|\mid \Lambda^{s} G_{q, t} \bar{\partial}_{b, t}^{*} g\right\|_{t}\left(\left\|\mid \Lambda^{s} \bar{\partial}_{b} G_{q, t} \bar{\partial}_{b, t}^{*} g\right\|_{t}+C_{t}\left\|\Lambda^{s-1} \bar{\partial}_{b} G_{q, t} \bar{\partial}_{b, t}^{*} g\right\| \|_{t}\right)\right) \\
& \leq\left|\left\langle\Lambda^{s} G_{q, t} \bar{\partial}_{b, t}^{*} g, \Lambda^{s} \bar{\partial}_{b, t}^{*} g\right\rangle_{t}\right|+\frac{1}{2}\left\|\Lambda^{s} \bar{\partial}_{b} G_{q, t} \bar{\partial}_{b, t}^{*} g\right\|_{t} \\
& \quad+O\left(\left\|\Lambda^{s} G_{q, t} \bar{\partial}_{b, t}^{*} g\right\|_{t}^{2}+C_{t}\left\|\mid \Lambda^{s-1} \bar{\partial}_{b} G_{q, t} \bar{\partial}_{b, t}^{*} g\right\|_{t}^{2}\right) .
\end{aligned}
$$

Thus, using induction in $s$ to estimate $\left\|\Lambda^{s-1} \bar{\partial}_{b} G_{q, t} \bar{\partial}_{b, t}^{*} g\right\|_{t}^{2}$,

$$
\left\|\left|\Lambda^{s} \bar{\partial}_{b} G_{q, t} \bar{\partial}_{b, t}^{*} g\right|\right\|_{t}^{2} \leq 2\left|\left\langle\Lambda^{s} G_{q, t} \bar{\partial}_{b, t}^{*} g, \Lambda^{s} \bar{\partial}_{b, t}^{*} g\right\rangle_{t}\right|+C \mid\left\|\Lambda^{s} G_{q, t} \bar{\partial}_{b, t}^{*} g\right\|_{t}^{2}+C_{t}\left\|\Lambda^{s-1} g\right\|_{t}^{2} .
$$

Next,

$$
\begin{aligned}
\left\langle\Lambda^{s} G_{q, t} \bar{\partial}_{b, t}^{*} g, \Lambda^{s} \bar{\partial}_{b, t}^{*} g\right\rangle_{t} & =\left\langle\Lambda^{s} \bar{\partial}_{b} G_{q, t} \bar{\partial}_{b, t}^{*} g, \Lambda^{s} g\right\rangle_{t} \\
& +O\left(\left\|\Lambda^{s} G_{q, t} \bar{\partial}_{b, t}^{*} g\right\|\left\|_{t}\right\| \mid \Lambda^{s} g\left\|_{t}+C_{t}\right\| \Lambda^{s} G_{q, t} \bar{\partial}_{b, t}^{*} g\|\|_{t}\left\|\Lambda^{s-1} g\right\|_{t}\right) .
\end{aligned}
$$

Thus, by absorbing terms after a small constant/large constant argument, we have

$$
\left\|\mid \Lambda^{s} \bar{\partial}_{b} G_{q, t} \bar{\partial}_{b, t}^{*} g\right\|_{t}^{2} \leq C\|\| \Lambda^{s} g\left\|_{t}^{2}+C\right\|\left\|\Lambda^{s} G_{q, t} \bar{\partial}_{b, t}^{*} g\right\|_{t}^{2}+C_{t}\left\|\Lambda^{s-1} g\right\|_{t}^{2}
$$

Finally, by choosing $\epsilon$ sufficiently small in (5) to absorb the $\left\|\Lambda^{s} G_{q, t} \bar{\partial}_{b, t}^{*} g\right\|_{t}^{2}$ terms, we have proven

$$
\|\| \Lambda^{s} G_{q, t} \bar{\partial}_{b, t}^{*} g\left\|_{t}^{2} \leq \epsilon\right\| \Lambda^{s} g\left\|_{t}^{2}+C_{t}\right\| \Lambda^{s-1} g \|_{t}^{2} .
$$

The argument to prove

$$
\left\|\Lambda^{s} G_{q, t} \bar{\partial}_{b} g\right\|_{t}^{2} \leq \epsilon\left\|\Lambda^{s} g\right\|_{t}^{2}+C_{t}\left\|\Lambda^{s-1} g\right\|_{t}^{2}
$$


is similar, the only difference being that $\bar{\partial}_{b, t}^{*}$ creates lower order terms that depend on $t$, but those are handled with the induction hypothesis and the $C_{t}\|\| \Lambda^{s-1} g \|_{t}^{2}$ term. This proves the proposition.

3.1. Proof of Proposition 2.3. Since $C_{0, q}^{\infty}(M)$ is dense in $W_{0, q}^{k}(M)$, it suffices to show the result for $u \in C_{0, q}^{\infty}(M)$. Our proof goes by induction. Since $M$ satisfies weak $Y(q)$, the $k=0$ case was proved in HR11. Assume that the result holds for all $\ell^{\prime}$ so that $0 \leq \ell^{\prime} \leq \ell \leq k-1$. Set $\Lambda_{\nu}^{s}=\tilde{\zeta}_{\nu} \Lambda^{s} \zeta_{\nu}$. Then

$$
\left\|\bar{\partial}_{b} G_{q} u\right\|_{W^{\ell+1}(M)}^{2}+\left\|\bar{\partial}_{b}^{*} G_{q} u\right\|_{W^{\ell+1}(M)}^{2}=\sum_{\nu}\left(\left\|\Lambda_{\nu}^{\ell+1} \bar{\partial}_{b} G_{q} u\right\|_{L^{2}(M)}^{2}+\left\|\Lambda_{\nu}^{\ell+1} \bar{\partial}_{b}^{*} G_{q} u\right\|_{L^{2}(M)}^{2}\right) .
$$

Examining one term from the sum (call it $R H S$ ), we first observe that $\bar{\partial}_{b}^{*} \Lambda_{\nu}^{\ell+1} \bar{\partial}_{b} G_{q} u$ and $\bar{\partial}_{b} \Lambda_{\nu}^{\ell+1} \bar{\partial}_{b}^{*} G_{q} u$ are both well-defined terms. For,

$$
\begin{aligned}
\Lambda_{\nu}^{\ell+1} u+\left[\bar{\partial}_{b}^{*}, \Lambda_{\nu}^{\ell+1}\right] & \bar{\partial}_{b} G_{q} u+\left[\bar{\partial}_{b}, \Lambda_{\nu}^{\ell+1}\right] \bar{\partial}_{b}^{*} G_{q} u \\
& =\Lambda_{\nu}^{\ell+1}\left(\bar{\partial}_{b} \bar{\partial}_{b}^{*}+\bar{\partial}_{b}^{*} \bar{\partial}_{b}\right) G_{q} u+\left[\bar{\partial}_{b}^{*}, \Lambda_{\nu}^{\ell+1}\right] \bar{\partial}_{b} G_{q} u+\left[\bar{\partial}_{b}, \Lambda_{\nu}^{\ell+1}\right] \bar{\partial}_{b}^{*} G_{q} u \\
& =\bar{\partial}_{b}^{*} \Lambda_{\nu}^{\ell+1} \bar{\partial}_{b} G_{q} u+\bar{\partial}_{b} \Lambda_{\nu}^{\ell+1} \bar{\partial}_{b}^{*} G_{q} u,
\end{aligned}
$$

so we can make sense of the right-hand side in terms of $\ell+2$ derivatives of $G_{q} u$ and $\ell+1$ derivatives of $u$, both well-defined quantities. We can use integration by parts to observe $R H S$ equals

$$
\begin{aligned}
& \left(\bar{\partial}_{b}^{*} \Lambda_{\nu}^{\ell+1} \bar{\partial}_{b} G_{q} u, \Lambda_{\nu}^{\ell+1} G_{q} u\right)_{0}+\left(\bar{\partial}_{b} \Lambda_{\nu}^{\ell+1} \bar{\partial}_{b}^{*} G_{q} u, \Lambda_{\nu}^{\ell+1} G_{q} u\right)_{0} \\
& \quad+O\left(\left\|\Lambda_{\nu}^{\ell+1} G_{q} u\right\|_{L^{2}(M)}\left(\left\|\Lambda_{\nu}^{\ell+1} \bar{\partial}_{b} G_{q} u\right\|_{L^{2}(M)}+\Lambda_{\nu}^{\ell+1} \bar{\partial}_{b}^{*} G_{q} u \|_{L^{2}(M)}\right)\right) \\
& =\left(\Lambda_{\nu}^{\ell+1} \square_{b} G_{q} u, \Lambda_{\nu}^{\ell+1} G_{q} u\right)_{0}+O\left(\left\|\Lambda_{\nu}^{\ell+1} G_{q} u\right\|_{L^{2}(M)}\left(\left\|\Lambda_{\nu}^{\ell+1} \bar{\partial}_{b} G_{q} u\right\|_{L^{2}(M)}+\left\|\Lambda_{\nu}^{\ell+1} \bar{\partial}_{b}^{*} G_{q} u\right\|_{L^{2}(M)}\right)\right) \\
& =\left(\Lambda_{\nu}^{\ell+1}\left(u-H_{q} u\right), \Lambda_{\nu}^{\ell+1} G_{q} u\right)_{0} \\
& +O\left(\left\|\Lambda_{\nu}^{\ell+1} G_{q} u\right\|_{L^{2}(M)}\left(\left\|\Lambda_{\nu}^{\ell+1} \bar{\partial}_{b} G_{q} u\right\|_{L^{2}(M)}+\left\|\Lambda_{\nu}^{\ell+1} \bar{\partial}_{b}^{*} G_{q} u\right\|_{L^{2}(M)}\right)\right) .
\end{aligned}
$$

Using a small constant/large constant argument and the fact that $H_{q} u=0$, we observe that

$$
\left\|\bar{\partial}_{b} G_{q} u\right\|_{W^{\ell+1}(M)}^{2}+\left\|\bar{\partial}_{b}^{*} G_{q} u\right\|_{W^{\ell+1}(M)}^{2} \leq C_{\ell+1}\left(\left\|G_{q} u\right\|_{W^{\ell+1}(M)}^{2}+\|u\|_{W^{\ell+1}(M)}^{2}\right) .
$$

For $\bar{\partial}_{b}^{*} \bar{\partial}_{b} G_{q} u$ and $\bar{\partial}_{b} \bar{\partial}_{b}^{*} G_{q} u$, we also use induction and an integration by parts argument. Since $\bar{\partial}_{b} \bar{\partial}_{b}^{*} \bar{\partial}_{b} G_{q} u=\bar{\partial}_{b}\left(\bar{\partial}_{b}^{*} \bar{\partial}_{b} G_{q}+\bar{\partial}_{b} \bar{\partial}_{b}^{*} G_{q}+H_{q}\right) u=\bar{\partial}_{b} u$, and $u \in W_{0, q}^{k+2}(M)$, it follows that $\left[\Lambda_{\nu}^{k}, \bar{\partial}_{b}\right] \bar{\partial}_{b}^{*} \bar{\partial}_{b} G_{q} u+\Lambda_{\nu}^{k} \bar{\partial}_{b} \bar{\partial}_{b}^{*} \bar{\partial}_{b} G_{q} u=\bar{\partial}_{b} \Lambda_{\nu}^{k} \bar{\partial}_{b}^{*} \bar{\partial}_{b} G_{q} \in W_{0, q}^{k+2}(M)$. For the induction, the $k=0$ case follows from [HR11. Assume that the result holds for all $\ell^{\prime}$ so that $0 \leq \ell^{\prime} \leq \ell \leq k-1$. Therefore, since $\ell+1 \leq k$,

$$
\begin{aligned}
& \left\|\Lambda_{\nu}^{\ell+1} \bar{\partial}_{b}^{*} \bar{\partial}_{b} G_{q} u\right\|_{L^{2}(M)}^{2} \\
& =\left(\bar{\partial}_{b} \Lambda_{\nu}^{\ell+1} \bar{\partial}_{b}^{*} \bar{\partial}_{b} G_{q} u, \Lambda_{\nu}^{\ell+1} \bar{\partial}_{b} G_{q} u\right)_{0}+O\left(\left\|\Lambda_{\nu}^{\ell+1} \bar{\partial}_{b}^{*} \bar{\partial}_{b} G_{q} u\right\|_{L^{2}(M)}\left\|\Lambda_{\nu}^{\ell+1} \bar{\partial}_{b} G_{q} u\right\|_{L^{2}(M)}\right) \\
& =\left(\Lambda_{\nu}^{\ell+1} \bar{\partial}_{b} u, \Lambda_{\nu}^{\ell+1} \bar{\partial}_{b} G_{q} u\right)_{0}+O\left(\left\|\Lambda_{\nu}^{\ell+1} \bar{\partial}_{b}^{*} \bar{\partial}_{b} G_{q} u\right\|_{L^{2}(M)}\left\|\Lambda_{\nu}^{\ell+1} \bar{\partial}_{b} G_{q} u\right\|_{L^{2}(M)}\right) \\
& =\left(\Lambda_{\nu}^{\ell+1} u, \Lambda_{\nu}^{\ell+1} \bar{\partial}_{b}^{*} \bar{\partial}_{b} G_{q} u\right)_{0} \\
& +O\left(\left\|\Lambda_{\nu}^{\ell+1} u\right\|_{L^{2}(M)}\left\|\Lambda_{\nu}^{\ell+1} \bar{\partial}_{b} G_{q} u\right\|_{L^{2}(M)}+\left\|\Lambda_{\nu}^{\ell+1} \bar{\partial}_{b}^{*} \bar{\partial}_{b} G_{q} u\right\|_{L^{2}(M)}\left\|\Lambda_{\nu}^{\ell+1} \bar{\partial}_{b} G_{q} u\right\|_{L^{2}(M)}\right) .
\end{aligned}
$$

Using a small constant/large constant argument and the earlier part of the argument, we may conclude that

$$
\left\|\bar{\partial}_{b}^{*} \bar{\partial}_{b} G_{q} u\right\|_{W^{\ell+1}(M)}^{2}+\left\|\bar{\partial}_{b}^{*} G_{q} u\right\|_{W^{\ell+1}(M)}^{2} \leq C_{\ell+1}\left(\left\|G_{q} u\right\|_{W^{\ell+1}(M)}^{2}+\|u\|_{W^{\ell+1}(M)}^{2}\right) .
$$

A similar argument shows the bound for $\bar{\partial}_{b} \bar{\partial}_{b}^{*} G_{q} u$. 
3.2. Proof of Theorem 2.1. The idea of the proof is simple: the results follow immediately by expressing $G_{q}$ in terms of $S_{q-1}, S_{q}, S_{q+1}^{\prime}$, and weighted operators (that we know are continuous on $W^{s}$ ) and, conversely, by expressing $S_{q-1}, S_{q}, S_{q+1}^{\prime}$ in terms of $G_{q}$ and weighted operators.

Let $s \geq 0$. From [HR11, we know that there exists $T_{s}$ so that if $t \geq T_{s}$, then all of the weighted operators: $G_{q, t}, \bar{\partial}_{b} G_{q, t}, \bar{\partial}_{b}^{*} G_{q, t}, G_{q, t} \bar{\partial}_{b}, G_{q, t} \bar{\partial}_{b}^{*}, I-\bar{\partial}_{b, t}^{*} \overline{\bar{\partial}}_{b} G_{q, t}, S_{q-1, t}$, $S_{q+1, t}^{\prime}$ are continuous in the $W^{s}$-norm on their respective spaces. The continuity of $I-\bar{\partial}_{b, t}^{*} \bar{\partial}_{b} G_{q, t}$ trivially gives continuity of $\bar{\partial}_{b, t}^{*} \bar{\partial}_{b} G_{q, t}$. Also, the argument in [HR11, Section 6.6] implies the continuity of $\bar{\partial}_{b} \bar{\partial}_{b, t}^{*} G_{q, t}$. Moreover, since

$$
H_{q, t}=I-\bar{\partial}_{b, t}^{*} \bar{\partial}_{b} G_{q, t}-\bar{\partial}_{b} \bar{\partial}_{b, t}^{*} G_{q, t},
$$

it follows that $H_{q, t}$ is continuous in $W_{0, q}^{s}(M)$. Finally, to show that $S_{q+1, t}^{\prime}$ is continuous in $W_{0, q+1}^{s}$, we note that $W_{0, q+1}^{s+1}(M)$ is dense in $W_{0, q+1}^{s}(M)$ and let $\varphi \in W_{0, q}^{s+1}(M)$. We then observe that

$$
\begin{aligned}
& \|\| \Lambda^{s} \bar{\partial}_{b} G_{q, t} \bar{\partial}_{b, t}^{*} \varphi \|_{t}^{2}=\left\langle\Lambda^{s} \bar{\partial}_{b, t}^{*} \bar{\partial}_{b} G_{q, t} \bar{\partial}_{b, t}^{*} \varphi, \Lambda^{s} G_{q, t} \bar{\partial}_{b, t}^{*} \varphi\right\rangle_{t} \\
& \quad+\left\langle\left[\bar{\partial}_{b, t}^{*}, \Lambda^{s}\right] \bar{\partial}_{b} G_{q, t} \bar{\partial}_{b, t}^{*} \varphi, \Lambda^{s} G_{q, t} \bar{\partial}_{b, t}^{*} \varphi\right\rangle_{t}+\left\langle\Lambda^{s} \bar{\partial}_{b} G_{q, t} \bar{\partial}_{b, t}^{*} \varphi,\left[\Lambda^{s}, \bar{\partial}_{b}\right] G_{q, t} \bar{\partial}_{b, t}^{*} \varphi\right\rangle_{t} .
\end{aligned}
$$

Since $\bar{\partial}_{b, t}^{*} \varphi$ is $\bar{\partial}_{b, t}^{*}$-closed, it follows that $\bar{\partial}_{b, t}^{*} \bar{\partial}_{b} G_{q, t} \bar{\partial}_{b, t}^{*} \varphi=\bar{\partial}_{b, t}^{*} \varphi$ so that

$$
\begin{aligned}
& \left\langle\Lambda^{s} \bar{\partial}_{b, t}^{*} \bar{\partial}_{b} G_{q, t} \bar{\partial}_{b, t}^{*} \varphi, \Lambda^{s} G_{q, t} \bar{\partial}_{b, t}^{*} \varphi\right\rangle_{t}=\left\langle\Lambda^{s} \bar{\partial}_{b, t}^{*} \varphi, \Lambda^{s} G_{q, t} \bar{\partial}_{b, t}^{*} \varphi\right\rangle_{t} \\
= & \left\langle\Lambda^{s} \varphi, \Lambda^{s} \bar{\partial}_{b} G_{q, t} \bar{\partial}_{b, t}^{*} \varphi\right\rangle_{t}+\left\langle\Lambda^{s} \varphi,\left[\bar{\partial}_{b}, \Lambda^{s}\right] G_{q, t} \bar{\partial}_{b, t}^{*} \varphi\right\rangle_{t}+\left\langle\left[\Lambda^{s}, \bar{\partial}_{b, t}^{*}\right] \varphi, \Lambda^{s} G_{q, t} \bar{\partial}_{b, t}^{*} \varphi\right\rangle_{t}
\end{aligned}
$$

It now follows that

$$
\left\|\Lambda^{s} \bar{\partial}_{b} G_{q, t} \bar{\partial}_{b, t}^{*} \varphi\right\|_{t}^{2} \leq C_{s, t}\left(\left\|\Lambda^{s} \varphi\right\|\left\|_{t}\right\| \Lambda^{s} \bar{\partial}_{b} G_{q, t} \bar{\partial}_{b, t}^{*} \varphi\left\|_{t}+\right\|\left\|\Lambda^{s} \varphi\right\|_{t}^{2}\right) .
$$

Using a small constant/large constant argument and absorbing terms, we have the continuity of $S_{q+1, t}^{\prime}$ in $W_{0, q+1}^{s}(M)$.

We now express $S_{q-1}, S_{q}$, and $S_{q+1}^{\prime}$ in terms of $G_{q}$. For $S_{q}$, continuity in $W^{s}$ follows from the formula

and Proposition 2.3 .

$$
S_{q}=I-\bar{\partial}_{b}^{*} \bar{\partial}_{b} G_{q}
$$

Assume that $G_{q}$ is exactly regular. Assume that $g$ is a $\bar{\partial}_{b}$-closed $(0, q-1)$-form. Then following [Str10, Section 5.3] (with the zero-order pseudodifferential operator $F_{t}$ defined in (4) replacing the weight), we have

$$
\begin{aligned}
\left(S_{q-1} f, g\right)_{0}=(f, g)_{0}=\left\langle F_{t}^{-1} f, g\right\rangle_{t} & =\left\langle S_{q-1, t} F_{t}^{-1} f, g\right\rangle_{t} \\
& =\left(F_{t} S_{q-1, t} F_{t}^{-1} f, g\right)_{0}=\left(S_{q-1} F_{t} S_{q-1, t} F_{t}^{-1} f, g\right)_{0} .
\end{aligned}
$$

Using the fact that $S_{q-1}=I-\bar{\partial}_{b}^{*} G_{q} \bar{\partial}_{b}$, it follows that

$$
\begin{aligned}
S_{q-1} & =S_{q-1} F_{t} S_{q-1, t} F_{t}^{-1}=\left(I-\bar{\partial}_{b}^{*} G_{q} \bar{\partial}_{b}\right) F_{t} S_{q-1, t} F_{t}^{-1} \\
& =F_{t} S_{q-1, t} F_{t}^{-1}-\bar{\partial}_{b}^{*} G_{q}\left[\bar{\partial}_{b}, F_{t}\right] S_{q-1, t} F_{t}^{-1} .
\end{aligned}
$$

For $S_{q+1}^{\prime}$, we first observe that by [HR11, (18)],

$\bar{\partial}_{b, t}^{*}\left(I-S_{q+1, t}^{\prime}\right)=\bar{\partial}_{b, t}^{*}-\bar{\partial}_{b, t}^{*} \bar{\partial}_{b} G_{q, t} \bar{\partial}_{b, t}^{*}=\bar{\partial}_{b} \bar{\partial}_{b, t}^{*} G_{q, t} \bar{\partial}_{b, t}^{*}+H_{q, t} \bar{\partial}_{b, t}^{*}=G_{q, t} \bar{\partial}_{b} \bar{\partial}_{b, t}^{*} \bar{\partial}_{b, t}^{*}=0$. 
Next, observe that $S_{q+1, t}^{\prime}=S_{q+1}^{\prime} S_{q+1, t}^{\prime}$, so we write

$$
\begin{aligned}
S_{q+1}^{\prime} & =S_{q+1, t}^{\prime}+S_{q+1}^{\prime}-S_{q+1}^{\prime} S_{q+1, t}^{\prime}=S_{q+1, t}^{\prime}+\bar{\partial}_{b} G_{q} \bar{\partial}_{b}^{*}\left(I-S_{q+1, t}^{\prime}\right) \\
& =S_{q+1, t}^{\prime}+\bar{\partial}_{b} G_{q}\left(\bar{\partial}_{b}^{*}-\bar{\partial}_{b, t}^{*}\right)\left(I-S_{q+1, t}^{\prime}\right) .
\end{aligned}
$$

We now express $G_{q}$ in terms of $S_{q-1}, S_{q}$, and $S_{q+1}^{\prime}$. We write

$$
G_{q}=G_{q}\left(\bar{\partial}_{b} \bar{\partial}_{b}^{*}+\bar{\partial}_{b}^{*} \bar{\partial}_{b}\right) G_{q}=\left(\bar{\partial}_{b}^{*} G_{q}\right)^{*}\left(\bar{\partial}_{b}^{*} G_{q}\right)+\left(G_{q} \bar{\partial}_{b}^{*}\right)\left(G_{q} \bar{\partial}_{b}^{*}\right)^{*} .
$$

Also, from [HR11, (22)], we know that if $\bar{\partial}_{b}^{*} \phi=0$, then $\bar{\partial}_{b}^{*} G_{q} \phi=0$, so $\bar{\partial}_{b}^{*}\left(I-S_{q}\right)=$ $\bar{\partial}_{b}^{*} \bar{\partial}_{b}^{*} \bar{\partial}_{b} G_{q}=0$ means that

$$
\begin{aligned}
\bar{\partial}_{b}^{*} G_{q} & =\bar{\partial}_{b}^{*} G_{q} S_{q}=\bar{\partial}_{b}^{*} G_{q}\left(\bar{\partial}_{b} \bar{\partial}_{b, t}^{*} G_{q, t}+\bar{\partial}_{b, t}^{*} \bar{\partial}_{b} G_{q, t}+H_{q, t}\right) S_{q} \\
& =\left(I-S_{q-1}\right) \bar{\partial}_{b, t}^{*} G_{q, t} S_{q}+\bar{\partial}_{b}^{*} G_{q} \bar{\partial}_{b, t}^{*} \underbrace{\bar{\partial}_{b} G_{q, t} S_{q}}_{=0}+\bar{\partial}_{b}^{*} G_{q} H_{q, t} S_{q} \\
& =\left(I-S_{q-1}\right) \bar{\partial}_{b, t}^{*} G_{q, t} S_{q}+\bar{\partial}_{b}^{*} G_{q} H_{q, t} S_{q} .
\end{aligned}
$$

Note that $S_{q} G_{q} \bar{\partial}_{b}^{*}=0$ since $\left(S_{q} G_{q} \bar{\partial}_{b}^{*}\right)^{*}=\bar{\partial}_{b} G_{q} S_{q}=0$. Also, $\bar{\partial}_{b}=S_{q} \bar{\partial}_{b}$ and $H_{q, t}=$ $S_{q} H_{q, t}$ since Range $\left(\bar{\partial}_{b}\right) \subset \operatorname{ker}\left(\bar{\partial}_{b}\right)$ and $\bar{\partial}_{b} H_{q, t}=0$, respectively. Consequently,

$$
\begin{aligned}
G_{q} \bar{\partial}_{b}^{*} & =\left(I-S_{q}\right) G_{q} \bar{\partial}_{b}^{*} \\
& =\left(I-S_{q}\right)\left[G_{q, t} \bar{\partial}_{b, t}^{*} \bar{\partial}_{b}+G_{q, t} \bar{\partial}_{b} \bar{\partial}_{b, t}^{*}+H_{q, t}\right] G_{q} \bar{\partial}_{b}^{*} \\
& =\left(I-S_{q}\right) G_{q, t} \bar{\partial}_{b, t}^{*} S_{q+1}^{\prime}+\left(I-S_{q}\right) G_{q, t} \bar{\partial}_{b} \bar{\partial}_{b, t}^{*} G_{q} \bar{\partial}_{b}^{*}+\left(I-S_{q}\right) H_{q, t} G_{q} \bar{\partial}_{b}^{*} \\
& =\left(I-S_{q}\right) G_{q, t} \bar{\partial}_{b, t}^{*} S_{q+1}^{\prime}+\underbrace{\left(I-S_{q}\right) S_{q}}_{=0} \bar{\partial}_{b} \bar{\partial}_{b, t}^{*} G_{q, t} G_{q} \bar{\partial}_{b}^{*}+\underbrace{\left(I-S_{q}\right) S_{q}}_{=0} H_{q, t} G_{q} \bar{\partial}_{b}^{*} \\
& =\left(I-S_{q}\right) G_{q, t} \bar{\partial}_{b, t}^{*} S_{q+1}^{\prime} .
\end{aligned}
$$

We also need to control $\left(\bar{\partial}_{b}^{*} G_{q}\right)^{*}$ and $\left(G_{q} \bar{\partial}_{b}^{*}\right)^{*}$. If $T_{t}$ is a continuous operator on $L^{2}\left(M, e^{-t|z|^{2}}\right)$, then we can compute its adjoint in $L^{2}(M)$ as follows:

$$
\left(T_{t} f, g\right)_{0}=\left\langle T_{t} f, F_{t}^{-1} g\right\rangle_{t}=\left\langle f, T_{t}^{*} F_{t}^{-1} g\right\rangle_{t}=\left(f, F_{t} T_{t}^{*} F_{t}^{-1} g\right)_{0},
$$

and we observe that the adjoint of $T_{t}$ is $F_{t} T_{t}^{*} F_{t}^{-1}$. We therefore compute

$$
\left(\bar{\partial}_{b}^{*} G_{q}\right)^{*}=F_{t} S_{q} G_{q, t} \bar{\partial}_{b}\left(I-S_{q-1}\right) F_{t}^{-1}+F_{t} S_{q} H_{q, t} G_{q} \bar{\partial}_{b} F_{t}^{-1}
$$

and

$$
\left(G_{q} \bar{\partial}_{b}^{*}\right)^{*}=F_{t} S_{q+1}^{\prime} \bar{\partial}_{b}\left(I-S_{q}\right) F_{t}^{-1} .
$$

We now investigate the harmonic projection $H_{q, t}$. From [HR11, p.156], we know that for a $(0, q)$-form $\varphi$,

$$
\|\varphi\|_{W^{s}(M)}^{2} \leq C_{t}\left(\left\|\bar{\partial}_{b} \varphi\right\|_{W^{s}(M)}^{2}+\left\|\bar{\partial}_{b, t}^{*} \varphi\right\|_{W^{s}(M)}^{2}+\|\varphi\|_{W^{s-1}(M)}^{2}\right) .
$$

By density, this means that for any $f \in L_{0, q}^{2}(M)$,

$$
\left\|H_{q, t} f\right\|_{W^{s}(M)}^{2} \leq C_{t, s}\left\|H_{q, t} f\right\|_{L^{2}(M)}^{2} \leq C_{t, s}\|f\|_{L^{2}(M)}^{2} .
$$

Therefore, if $t \geq T_{s+1}$, then $H_{q, t}: L_{0, q}^{2}(M) \rightarrow W_{0, q}^{s+1}(M)$ and

$$
\begin{aligned}
& \left\|\bar{\partial}_{b}^{*} G_{q} H_{q, t} S_{q} f\right\|_{W^{s}(M)} \leq C\left\|G_{q} H_{q, t} S_{q} f\right\|_{W^{s+1}(M)} \\
& \quad \leq C\left\|H_{q, t} S_{q} f\right\|_{W^{s+1}(M)} \leq C_{s, t}\left\|H_{q, t} S_{q} f\right\|_{L^{2}(M)} \leq C_{s, t}\|f\|_{L^{2}(M)},
\end{aligned}
$$

so $\bar{\partial}_{b}^{*} G_{q} H_{q, t} S_{q}: L_{0, q}^{2}(M) \rightarrow W_{0, q-1}^{s}(M)$. 


\section{Stein manifolds}

Finally, we briefly indicate how to adapt the argument to prove our main result in the case of a Stein manifold. We need the following result.

Theorem 4.1. Let $M$ be an n-dimensional Stein manifold and $\Omega \subset M$ be a bounded subset with a smooth boundary satisfying weak $Z(q)$ for some $1 \leq q \leq n-1$. Then there exists $\tilde{t}>0$ such that for all $t>\tilde{t}$ and $s \geq-\frac{1}{2}$ we have:

(1) The weighted $\bar{\partial}-$ Neumann operator $N_{q, t}$ exists and is continuous in $W_{0, q}^{s}(\Omega)$.

(2) The canonical solution operators to $\bar{\partial}$ given by

$$
\bar{\partial}_{t}^{*} N_{t}^{q}: W_{0, q}^{s}(\Omega) \rightarrow W_{0, q-1}^{s}(\Omega) \text { and } N_{t}^{q} \bar{\partial}_{t}^{*}: W_{0, q+1}^{s}(\Omega) \rightarrow W_{0, q}^{s}(\Omega)
$$

are continuous.

(3) The canonical solution operators to $\bar{\partial}_{t}^{*}$ given by

$$
\bar{\partial} N_{t}^{q}: W_{0, q}^{s}(\Omega) \rightarrow W_{0, q+1}^{s}(\Omega) \text { and } N_{t}^{q} \bar{\partial}: W_{0, q-1}^{s}(\Omega) \rightarrow W_{0, q}^{s}(\Omega)
$$

are continuous.

(4) For every $f \in W_{0, q}^{s}(\Omega) \cap \operatorname{ker} \bar{\partial}$ there exists $u \in W_{0, q-1}^{s}(\Omega)$ such that $\bar{\partial} u=f$.

In [HR], Harrington and Raich proved Theorem 4.1 for $-\frac{1}{2} \leq s \leq 1$. Standard techniques show that their arguments extend seamlessly to all $s \geq-\frac{1}{2}$.

The proofs of the results in Section 2.2 are now straightforward, given the proofs of Section 2.1 and [Str10, Section 5.3]. The general outline of the argument is contained in [Str10, Section 5.3]. Our hypotheses allow us to prove closed range and Kohn's weighted theory for a fixed $q, 1 \leq q \leq n-1$. Using the arguments from the proofs of the results in Section 2.1 with the weighted theory from Theorem 4.1. $L^{2}$ theory from $[\mathrm{HR}$, and the recognition that the tangential derivatives control the Sobolev norms (so we can replace the $\Lambda^{k}$ terms with $D_{T^{\alpha}}$ ), we can repeat the arguments above to prove the results in Section 2.2 ,

\section{REFERENCES}

[ABZ06] Heungju Ahn, Luca Baracco, and Giuseppe Zampieri, Non-subelliptic estimates for the tangential Cauchy-Riemann system, Manuscripta Math. 121 (2006), no. 4, 461-479, DOI 10.1007/s00229-006-0049-z. MR2283474 (2007j:32042)

[Bar12a] Luca Baracco, Erratum to: The range of the tangential Cauchy-Riemann system to a CR embedded manifold [MR2981820], Invent. Math. 190 (2012), no. 2, 511-512, DOI 10.1007/s00222-012-0425-0. MR2981821

[Bar12b] Luca Baracco, The range of the tangential Cauchy-Riemann system to a CR embedded manifold, Invent. Math. 190 (2012), no. 2, 505-510, DOI 10.1007/s00222-012-0387-2. MR 2981820

[Bog91] Albert Boggess, CR manifolds and the tangential Cauchy-Riemann complex, Studies in Advanced Mathematics, CRC Press, Boca Raton, FL, 1991. MR1211412 (94e:32035)

[BS86] Harold P. Boas and Mei-Chi Shaw, Sobolev estimates for the Lewy operator on weakly pseudoconvex boundaries, Math. Ann. 274 (1986), no. 2, 221-231, DOI 10.1007/BF01457071. MR838466 (87i:32029)

[BS90] Harold P. Boas and Emil J. Straube, Equivalence of regularity for the Bergman projection and the $\bar{\partial}-$ Neumann operator, Manuscripta Math. 67 (1990), no. 1, 25-33, DOI 10.1007/BF02568420. MR.1037994 (90k:32057)

[CS01] So-Chin Chen and Mei-Chi Shaw, Partial differential equations in several complex variables, AMS/IP Studies in Advanced Mathematics, vol. 19, American Mathematical Society, Providence, RI, 2001. MR 1800297 (2001m:32071) 
[FK72] G. B. Folland and J. J. Kohn, The Neumann problem for the Cauchy-Riemann complex: Annals of Mathematics Studies, No. 75, Princeton University Press, Princeton, N.J., 1972. MR 0461588 (57 \#1573)

[Hör65] Lars Hörmander, $L^{2}$ estimates and existence theorems for the $\bar{\partial}$ operator, Acta Math. 113 (1965), 89-152. MR0179443(31 \#3691)

[HR] P. Harrington and A. Raich, Closed range for $\bar{\partial}$ and $\bar{\partial}_{b}$ on bounded hypersurfaces in Stein manifolds, submitted. arXiv:1106.0629.

[HR11] Phillip S. Harrington and Andrew Raich, Regularity results for $\bar{\partial}_{b}$ on CR-manifolds of hypersurface type, Comm. Partial Differential Equations 36 (2011), no. 1, 134-161, DOI 10.1080/03605302.2010.498855. MR2763350 (2012c:32048)

[Koh73] J. J. Kohn, Global regularity for $\bar{\partial}$ on weakly pseudo-convex manifolds, Trans. Amer. Math. Soc. 181 (1973), 273-292. MR0344703 (49 \#9442)

[Koh86] J. J. Kohn, The range of the tangential Cauchy-Riemann operator, Duke Math. J. 53 (1986), no. 2, 525-545, DOI 10.1215/S0012-7094-86-05330-5. MR850548 (87m:32041)

[Nic06] Andreea C. Nicoara, Global regularity for $\bar{\partial}_{b}$ on weakly pseudoconvex CR manifolds, Adv. Math. 199 (2006), no. 2, 356-447, DOI 10.1016/j.aim.2004.12.006. MR2189215 (2006h:32034)

[Rai10] Andrew Raich, Compactness of the complex Green operator on CR-manifolds of hypersurface type, Math. Ann. 348 (2010), no. 1, 81-117, DOI 10.1007/s00208-009-0470-1. MR 2657435 (2011i:32037)

[RS08] Andrew S. Raich and Emil J. Straube, Compactness of the complex Green operator, Math. Res. Lett. 15 (2008), no. 4, 761-778, DOI 10.4310/MRL.2008.v15.n4.a13. MR:2424911(2009i:32044)

[Sha85] Mei-Chi Shaw, $L^{2}$-estimates and existence theorems for the tangential Cauchy-Riemann complex, Invent. Math. 82 (1985), no. 1, 133-150, DOI 10.1007/BF01394783. MR808113 (87a:35136)

[Str10] Emil J. Straube, Lectures on the $\mathcal{L}^{2}$-Sobolev theory of the $\bar{\partial}$-Neumann problem, ESI Lectures in Mathematics and Physics, European Mathematical Society (EMS), Zürich, 2010. MR2603659 (2011b:35004)

[Zam08] Giuseppe Zampieri, Complex analysis and CR geometry, University Lecture Series, 43, Amer. Math. Soc., Providence, RI, 2008. MR2400390(2009f:32052)

Department of Mathematical Sciences, SCEn 301, University of Arkansas, FayetteVille, Arkansas 72701

E-mail address: psharrin@uark.edu

Departimento di Matematica, Università degli Studi di Milano, Via C. Saldini 50, 20133 Milano, Italy

E-mail address: marco.peloso@unimi.it

Department of Mathematical Sciences, SCEn 301, University of Arkansas, FayetteVILLE, Arkansas 72701

E-mail address: araich@uark.edu 